\title{
INFLUENCIA DE LOS HÁBITOS COMUNICATIVOS EN LA GENERACIÓN DE PARTICIPACIÓN Y CINISMO POLÍTICO ENTRE LOS PRECIUDADANOS
}

\section{Influence of Communicative Habits in Generating Participation and Political Cynicism among the Pre-Citizens}

\section{Influência dos hábitos comunicativos na geração de participação e cinismo político entre os pré-cidadãos}

SALDIERNA SALAS, Alma Rosa. Universidad Autónoma de Nuevo León (México) alma.saldiernas@uanl.mx

MUÑIZ, Carlos. Universidad Autónoma de Nuevo León (México)

carlos.munizm@uanl.mx

\section{Fecha de recibido: 31 de diciembre de 2014}

Fecha de aceptado: 2 de junio de 2015

\section{RESUMEN}

El presente trabajo analiza la influencia de los hábitos comunicativos en la generación de participación y cinismo político en los preciudadanos. Definiéndolos como aquellos jóvenes que por primera vez ejercerán el derecho a votar. El análisis se realiza desde la perspectiva de la teoría del malestar mediático y el círculo virtuoso. Los datos se obtuvieron a través de una encuesta aplicada a 1030 estudiantes del último año de preparatoria de la zona metropolitana de Monterrey, en el estado mexicano de Nuevo León. Para el análisis de datos se utilizó el programa 
SPSS versión 21. Entre los principales hallazgos se observa que el medio de comunicación que más consumen es la Internet seguido por la televisión, pero el uso es solo con fines de entretenimiento y no con fines de búsqueda de información política. Así mismo, se encuentra evidencia de que los niveles de conversación política mantenida por los preciudadanos impactan en actitudes como la participación y el cinismo político. Finalmente, se ha detectado que el tipo de medio consumido y la atención a ciertos contenidos no contribuyen al cambio en los niveles de participación política, contrario a la evidencia teórica precedente.

Palabras clave: hábitos comunicativos, participación ciudadana, cinismo político, preciudadanos.

\section{ABSTRACT}

The analysis focuses on the biasing effect of the communicative habits in generating participation and political cynicism in pre-citizens. Defining them as young people who first exercise the right to vote. It is approached from the perspective of the theory of media malaise and virtuous circle. Data were collected through a survey of 1030 seniors in high school in the metropolitan area of Nuevo Leon. For data analysis SPSS version 21. The main findings have used the media to consume more is the internet followed by television, but consumption is only for entertainment purposes as the fact chatting or downloading music not for purposes of seeking political information, also is evidence regarding their levels of conversation, these are really impacting attitudes and political cynicism. Furthermore, with regard to political participation is that the average consumed and attention to certain content are not contributing to the generation of this attitude, contrary to theoretical evidence.

Keywords: Communicative habits, political participation, political cynicism, pre-citizens.

\section{RESUMO}

O presente trabalho analisa a influência dos hábitos comunicativos na geração de participação e cinismo político nos pré-cidadãos. Definindo-os como aqueles jovens que por primeira vez exercerão o direito a votar. A análise se realiza desde a perspectiva da teoria do mal-estar mediático e o círculo virtuoso. Os dados obtiveram-se através de uma enquete aplicada a estudantes do último ano de ensino secundário da zona metropolitana de Monterrey, no estado mexicano de Nuevo León. Entre os principais achados observa-se que o meio de comunicação que mais consumem é a internet seguido pela televisão, mas o consumo é só com fins de entretenimento e não com fins de procura de informação política. Igualmente, encontra-se evidência de que os níveis de conversação política mantida pelos pré-cidadãos impactam em atitudes como a participação e o cinismo político. Finalmente, se tem detectado que o tipo de meio consumido e a atenção a certos conteúdos não contribuem à mudança nos níveis de participação política, contrário à evidência teórica precedente.

Palavras-chave: Hábitos comunicativos, participação cidadã, cinismo político, pré-cidadãos. 


\section{Introducción}

\section{Medios de comunicación y cambio de actitudes políticas}

Dentro de los estudios realizados desde la comunicación política, un tema ampliamente analizado es la influencia que tienen los medios de comunicación en la generación de un compromiso ciudadano con la política. En concreto, los estudios se han centrado en el papel preponderante que los medios de comunicación ejercen a la hora de generar corrientes de opinión y posturas ante el mundo político. En este sentido, los medios de comunicación constituyen una herramienta fundamental en la organización del Estado para conseguir y/o mantener el compromiso político de los ciudadanos (García-Luengo \& Maurer, 2009). Al llegar a una gran parte de la población, los medios de comunicación pueden influir y determinar las agendas de temas que debate la opinión pública y generar ciertas modas o modificar ideologías. Contribuyen a entablar relaciones entre el sector político y la sociedad (Avery, 2009) y facilitan la formación de un criterio de una actitud política en los individuos (Rojas, 2006).

De ellos, quizá la televisión sea la principal fuente de información política así como de formación de opinión pública, constituyendo por tanto una herramienta clave en el proceso de educación política (Sartori, 2005). En ese sentido, se considera que en una democracia ideal los medios reciben el respaldo de la ciudadanía al cumplir tres funciones claves para que se preserve la transparencia y confianza en ellos (Guerrero, 2006), que provean a la ciudadanía información suficiente para que sus miembros sean capaces de fundamentar sus elecciones, opiniones y decisiones. i) Los medios pueden verse como una fuente de recurso educativo, informativo y formativo. ii) Ellos ofrecen espacios donde se puede generar el debate abierto. iii) Son el ojo vigilante a favor de la ciudadanía, lo que se conoce como "el cuarto poder protector".

En los ultimos años se ha entablado un debate con el fin de entender los efectos políticos que el consumo de los medios tiene en la ciudadanía, diferenciando entre la simple exposición genérica a los mismos y la atención a sus contenidos. Así mismo, se ha mencionado el hecho de que no solo es la forma en que presentan la información, sino también el tipo de medio el que influye en la generación de esa apatía hacia la política, afirmando que la televisión tiene esa facultad a diferencia de los medios impresos (Newton 1999; Voltmer, 2007). Al respecto, se ha encontrado una clara diferencia entre los que simplemente están expuestos a los contenidos de manera general y los que centran su atención a contenidos en especifico de política (Prior, 2005), siendo mayor el impacto generado por la atención especializada a contenidos de los medios sobre política. Sin embargo, la evidencia empírica existente sobre el impacto que el consumo de medios de comunicación ejerce sobre las actitudes políticas como manifestación de la cultura política de una sociedad, presenta conclusiones diferenciadas.

Desde una perspectiva, se plantea cómo el uso de los medios contribuye a generar un aumento del cinismo político y una apatía ciudadana, el conocido como Malestar Mediático o Videomalestar (Schreiber \& García, 2003). El efecto se encuentra fuertemente relacionado con el consumo de televisión a diferencia de los medios impresos (Newton, 1999; Voltmer, 2007). Con respecto a los tipos de contenidos, existe evidencia de los efectos negativos del consumo de programas de entretenimiento en los niveles de participación política de los ciudadanos (Holtz-Bacha, 1990). Rojas (2006) refuerza esa evidencia, en la influencia en actitudes políticas como el conocimiento político, la participación cívica y los sentimientos de eficacia política. Un planteamiento consistente con lo evidenciado en el 
estudio de Shah, McLeod y Yoon (2001), quienes encuentran una influencia erosionadora de la actividad política entre aquellos que tienen elevado consumo mediático de contenidos de entretenimiento y diversión. Este paradigma se ha analizado principalmente en el caso de Estados Unidos (Capella \& Jamieson, 1997; de Vreese, 2005), pero también hay autores que han trabajado en el contexto europeo al respecto. Es el caso de Newton (1999), quien descubre esta relación en Inglaterra, así como Voltmer y Schmitt-Beck (2007) en Europa del este.

Por otro lado, otros autores han encontrado el efecto contrario, concluyendo que los contenidos de los medios son los que generan la movilización política de los ciudadanos. Es el caso de Pippa Norris (1996), quien considera que el consumo de noticias y televisión sobre asuntos políticos genera un aumento considerable en la participación política de los individuos. La autora detecta que los consumidores usuales de noticias en televisión, así como los lectores frecuentes de prensa informativa, muestran más interés en continuar informados, interesados y comprometidos con la vida política, generándose un círculo virtuoso (Norris, 2000). Ese efecto también se puede encontrar en medios de comunicación tradicionales como la televisión, radio prensa y últimamente el Internet (Stromback \& Shehata, 2010). Con respecto al Internet se puede mencionar que debido a la facilidad de acceder casi en tiempo real a través de comentarios y críticas, así como contenido satírico con respecto a las acciones realizadas por los políticos, este medio permite de manera más fácil participar en temas de política e involucrar y hacer partícipe al ciudadano del espacio virtual que tiene la política en la red (Anduiza, Cantijoch, Clelia, Gallego, \& Salcedo, 2010).

Así mismo, se ha puesto de manifiesto la necesidad de analizar los factores individuales y estructurales de los individuos para determinar los efectos de los medios en la generación de un comportamiento político. En ese sentido, Rojas (2006) explora, junto al rol de los medios de comunicación, el papel jugado por la conversación política, planteando como hipótesis que el consumo de noticias y el diálogo sobre estos temas se relaciona fuertemente con la participación política de los individuos. El autor también hace cuestionamientos sobre los efectos negativos de ciertas formas de entretenimiento. La evidencia que encuentra Rojas (2006) le lleva a concluir que la conversación política impacta en los procesos de participación política, pero no solo la interpersonal sino también la mediada a través de las nuevas tecnologías de comunicación como fuentes de información e interrelación personal. En concordancia, Newton (2006) afirma que los medios impactan en los ciudadanos, pero ese efecto es el resultado de la interacción entre los medios y otras fuerzas sociales que ejercen un efecto mediador en los efectos mediáticos, analizando también los niveles de conversación sobre política (Newton, 2006). Esto es, las relaciones personales tienen un impacto significativo sobre la probabilidad de un individuo en participar en actividades relacionadas a la política, tales como ir a votar (Schmitt-Beck \& Mckenrodt, 2010).

La participación política puede definirse como toda actividad de los ciudadanos dirigida a intervenir en la elección de sus gobernantes (Conway, 1986). Es un conjunto de actos y de actitudes que buscan "influir de manera más o menos directa y más o menos legal sobre las decisiones de los detentadores del poder en el sistema político o en cada una de las organizaciones políticas, así como en su misma selección", todo ello a fin de conservar o modificar la estructura y los valores del sistema de intereses que domina en la sociedad (Pasquino, 1994, p. 25). Es aquella acción de los ciudadanos que buscan influir en los resultados de los procesos políticos como las elecciones a cargos públicos, aplicación y formulación de políticas públicas, manifestaciones (Anduiza \& Bosch, 2004). Sin la participación de los ciudadanos en el proceso político la democracia carece de sentido y de legitimidad.

Para algunos autores como Norris (2000), la participación política se puede dividir en i) tradicional o convencional (voto, afiliación política, participación en campañas políticas, debates, etc.) esto es, actividades de apoyo al 
régimen. ii) no convencional (manifestaciones, movilizaciones. iii) Participación socio-política (movimientos sociales de oportunidad, no hay una jerarquía, existe una relación igualitaria en los participantes, es más bien dirigido a movimientos como protestas, o manifestaciones que podrían verse como actos contra el régimen). En ese sentido, se encuentran actividades totalmente relacionadas con el sistema político con lo cual el involucrarse en estas es la denominada participación política; pero también existen actividades condicionadas a niveles de compromiso por parte de los ciudadanos sin tener una implicación política como tal, ejemplo de esto es la participación en agrupaciones como clubs, manifestaciones, voluntariado, actividades más bien de compromiso social a lo que denomina participación cívica (Shah, Cho, Nah, Gotlieb, Lee, Scholl, Hwang, \& McLeod, 2007). Son los sentimientos y acciones emprendidas para resolver problemas, tener la oportunidad de participar en situaciones de mejora de la comunidad, de apoyo mutuo, etc. (Sirkku, 2009). La participación cívica tiene una implicación social que hace referencia a los hábitos de colaboración entre los ciudadanos (López, 2007), esto puede considerarse un ejemplo de la cultura cívica, ya que consiste en adoptar un compromiso solidario en la búsqueda del bien público (Cordourier, 2008).

Sin embargo, una de las manifestaciones de la falta de participación viene reflejada por la apatía o indiferencia de los ciudadanos hacia el sistema político, que ha sido conceptualizada como cinismo político. El cinismo político es, por tanto, explicado como la falta de confianza en las instituciones y entes políticos, representa por tanto una nula confianza, en las instituciones, gobierno y el sistema como un todo (Niemi, 1999), desencadenando una crisis de la democracia liberal representativa. Los ciudadanos perciben al sistema político como corrupto por lo que no desean involucrarse, pues participar sería para ellos convertirse en corruptos también (Capella \& Jamieson, 1997). Por su parte, Mariano Torgal (2000) considera que el cinismo político es un sentimiento generado por la ineficacia y la desafección política, generando un distanciamiento y alineación sobre el sistema político. El cinismo político se define también como el sentimiento de que los asuntos públicos son vistos como algo lejano, falto de importancia o carente de sentido. Incapacidad para influir en el sistema, y que el sistema, a su vez, responda a las demandas de los ciudadanos (Torgal, 2000).

Estas actitudes políticas se conforman a través de diversos elementos en el proceso de vida de los individuos, influenciadas por el medioambiente, sus compañeros de trabajo así como los medios de comunicación (Martínez \& Salcedo, 2006). Con respecto a los medios de comunicación, estos ejercen en los sistemas democráticos un rol informador, a la par de controlador, pues permiten conocer el ejercicio de los gobernantes y transmitirlo a la población en general y de esa manera contribuir en la formación de pautas de comportamiento en los individuos en su relación con el gobierno (Newton, 2006). Pueden ser fuente de información, así como contribuir a la generación de una opinión pública que ayude a promover la actividad política (Abundis, 2007).

Se ha encontrado evidencia respecto al importante impacto que el tipo de medio consumido tiene en la fuerza y la dirección del compromiso cívico de la ciudadanía, así como de distintos motivos personales que llevan al ciudadano a consumirlos y procesar sus contenidos (Norris, 2000). Algunos antecedentes se pueden evidenciar en los estudios de Lang y Lang (1966), quienes ya afirmaban la existencia de una desconexión sobre la política, una apatía; generada a traves del estilo que utilizan los noticieros para presentar las noticias sobre política. Por otro lado, y con respecto a la generación de participación, se ha detectado que los ciudadanos al estar expuestos de manera consistente a noticias en general suelen estar más documentados en términos políticos y, por lo tanto, confían en mayor medida en el gobierno y en el sistema político, contribuyendo a que sean más participativos (García Luengo, 2002). 
Al respecto de esta relación entre consumo mediático y actitudes políticas, Verba, Schlozman y Brady (1995) consideran que existe una causalidad recíproca entre comunicación y participación, esto es, que participar en política propicia que una persona hable en el futuro de política y viceversa, generándose una espiral en la relación entre comunicación y participación. Ello desembocaría en la formación de una corriente de información política que demuestra el interés en la política por parte de los ciudadanos, creando además un mayor compromiso cívico por parte de estos. Autores como Holtz-Bacha (1990), en un análisis realizado con datos panel, encuentran que la exposición a noticias en televisión y en periódicos no está asociado al videomalasie, sino que más bien una alta exposición a consumos de entretenimiento generan bajos niveles de participación política. Por tanto, no solo es la exposición a los medios de comunicación, sino también el tipo de programa consumido lo que impacta en el compromiso cívico de los ciudadanos.

Con todo, en la formación de ciudadanos y generación de compromiso con respecto a la política no solo influyen los medios de comunicación, sino que otras actividades comunicativas como la conversación sobre el tema permiten la generación de reflexiones acerca de política. En este sentido, este tipo de variables relacionadas con el diálogo político también podrían reforzar o modificar opiniones y comportamientos. La evidencia indica que la integración social y la participación cívica está asociada a elevados niveles de conversación política por parte de los individuos (McLeod, Scheufele, \& Moy, 1999). De hecho, autores como Rojas (2006) introducen esas variables a sus estudios, encontrando resultados consistentes de la importancia de la conversación sobre política para la generación de participación cívica y política.

\section{Los preciudadanos y su relación con la política en el caso mexicano}

Si bien la literatura presente demuestra el especial papel jugado por los diferentes hábitos comunicativos en la conformación de las actitudes políticas ciudadanas, cabe preguntarse por el papel que pueden jugar en el desarrollo de una cultura política entre los miembros de la sociedad que, debido a su edad, aún no han alcanzado el pleno ejercicio de sus derechos ciudadanos. Es por ello que el objeto de estudio del presente trabajo se encuentra focalizado en los jóvenes que por primera vez se enfrentaron al juego político de participar en unas elecciones presidenciales. Estos sujetos han sido denominados en esta investigación como preciudadanos, siguiendo la propuesta de otros autores que definen así a los jóvenes que están en el proceso de obtención de información sobre política para poder, por primera vez, ser parte del escenario político (García \& Micco, 1997).

En un trabajo de análisis de la formación de los ciudadanos desde edades tempranas, Conell (1971) define diversas etapas con el objeto de identificar los patrones que permitan determinar las nociones que tienen los niños sobre política. En sus resultados, el autor afirma que los niños inician el proceso de entender el entorno político en el que se encuentran inmersos entre los 9 y 12 años. Es en este momento cuando comienzan a emitir juicios que formarán parte de su cultura política. Sin embargo, es a partir de los 18 años cuando perciben claramente el papel que juegan como entes con la capacidad y el derecho de ser elementos activos del sistema político al que pertenecen. Es la etapa en la que los niños están en el proceso de exploración, adquisición, reflexión y que tienen pleno conocimiento y conciencia de su entorno como sujetos políticos (Coto, 1970). Ello pone de manifiesto la importancia de analizar la influencia de los diferentes factores, como los comunicativos, en los preciudadanos de cara a conformar sus actitudes políticas. Ya que es en esta edad cuando se encuentran en el proceso de aprendizaje para ser ciudadanos activos 
y conscientes de sus obligaciones dentro del juego político, o por el contrario de convertirse en ciudadanos indiferentes al proceso de toma de decisiones políticas.

En el caso de México, el Instituto Nacional de Estadística y Geografía en su último censo (INEGI, 2010) refleja que el 36,2 \% de la población se encuentra entre los 12 y 29 años, lo que representa la tercera parte de la población total del país. Así mismo, 11 millones de ellos contaban con entre 15 y 19 años. Por tanto, un número significativo de la población se encuentra en la etapa de la vida donde se comienzan a tomar decisiones que repercutirán en su futuro. Por su parte, los datos reflejan que Nuevo León, donde se realizó el estudio que da lugar al presente artículo, es un estado joven ya que el $27,7 \%$ de su población se encuentra entre los 0 y 14 años, así como un 26,6\% tienen entre 15 y 29 años. Estas cifras muestran el alcance que puede llegar a tener este sector de la población, ya que constituyen un segmento que está en proceso de desarrollo y es previsible que en el futuro tengan una fuerte influencia en el crecimiento y estabilidad económica, social y, por supuesto, política de México. Un ejemplo de ello es el caso de la pasada campaña para la Presidencia de la República de julio de 2012, donde se situaron en juego un total de 8 millones de votos correspondientes a los jóvenes que por primera vez participarían en una elección (IFE, 2012).

Con respecto a los niveles de desencanto y apatía sobre temas relacionados con la política, de acuerdo a la Encuesta Nacional de Juventud (IMJ, 2005), el 44,1\% de la población juvenil mexicana tiene un total desinterés por la política, el 39,8\% tiene poco interés con respecto a temas políticos, así como que atribuyen ese poco interés a que tienen desconfianza en los políticos (38,8\%). Al respecto de los datos del Estado de Nuevo León, la participación política es vista por una importante parte de los jóvenes como una acción centrada únicamente al voto en las elecciones (56,2\%), el 43,1\% de los encuestados cree que la única forma de participar es votando y el $50 \%$ no piensa participar en política electoral. Este resultado es consistente en la medición de 2012, en el que el 89,6\% de los participantes en el estudio afirmaron no estar interesados en la política del país además de reprobar a los partidos políticos y a los funcionarios. Unos resultados relativos a los jóvenes que ya forman parte del sistema por ser mayores de edad.

Pero ¿qué ocurre con aquellos más jóvenes que aún son preciudadanos?, aquellos que se encuentran aún en el proceso de aprendizaje y entenidimiento de las cuestiones relacionadas a la política. Los resultados de la Consulta Infantil realizada en abril de 2012 por el Instituto Federal Electoral permiten configurar un panorama sobre lo que están viviendo los niños de 6 a 15 años de edad. Los principales hallazgos de esta consulta muestran que la mayor participación se da entre los niños de entre 8 y 11 años, lo que representó el 50,2\% del total, siendo las niñas las que más acudieron a emitir sus ideas. Con respecto a la confianza en las instituciones, la familia, los maestros y el ejército coninciden en ser los que más confianza reciben de estos niños, un resultado similar el de los jóvenes mayores de 18 años antes mencionados. Esto ejemplifica cómo se presenta la confianza en la instituciones primarias, y esta parece trasladarse de unas edades o grupos a otros.

En lo que respecta a los niveles de participación de los niños encuestados en la citada Consulta Infantil, se observa cómo el factor inseguridad era importante. Se pudo constatar que en estados donde la inseguridad no era un problema diario o tan cercano, ese valor era superior a la media. Es decir, en estos estados los niños consideraban que podían elegir de forma efectiva a sus gobernantes y participar en la toma de decisiones, lo que pone de manifiesto cómo estos jóvenes cuentan con un nivel alto de eficacia política. Por el contrario, en los estados donde la inseguridad es muy fuerte, como era el caso de Nuevo León en 2012, ese dato estaba por debajo de la 
media. Es decir, los niños de estos estados presentaban un sentimiento de menor eficacia política al no creer que pudieran incidir en la toma de decisiones del sistema, lo que puede derivar en un aumento de su cinismo político. Este resultado refleja cómo el entorno de los niños está fuertemente ligado a la percepción que estos tengan sobre su participación en el juego político.

El informe realizado en 2012 por el Colegio de México (COLMEX) a nivel nacional arrojó datos similares. A partir de una encuesta realizada a 3250 jóvenes de 18 a 29 años, se detectó el hecho de que el $32 \%$ no contaba con una ideología política claramente establecida. Por otro lado, con respecto a su participación política, el $86 \%$ consideraba que el voto era poco o nada efectivo para presionar a las autoridades. Además, en lo que respecta a la participación ciudadana, entre el 90 y $97 \%$ jamás había formado parte de organizaciones culturales, estudiantiles, deportivas, religiosas, de partidos políticos o sindicatos (Gómez, Tejera, \& Aguilar, 2012). Estos resultados evidencian la apatía y la falta de interés en participar en la política por parte de los miembros más jóvenes de la sociedad, incluso en algo tan esencial para la vida democrática como es el hecho de emitir un voto en las elecciones. Esto los hace autodiscriminarse, puesto que aunque están en el proceso de construir su propia identidad, de formarse una personalidad y de ser escuchados, no parecen estar tomando las riendas para conseguir ser tenidos en cuenta de manera efectiva.

Los datos anteriormente presentados muestran que los jóvenes no están interesados en involucrarse en la política. Parece ser más bien que estos jóvenes se encuentran inmersos en su diversión y satisfacción personal, pero sin buscar repercutir en el engranaje socio-político del que son parte. Se quejan de lo que sucede, pero no parecen tomar medidas para mejorar la situación de su país, quizá porque no se han dado cuenta de la importancia que tienen sus acciones en el juego político de su nación. Su socialización se circunscribe en gran medida a temas de diversión, puesto que no demuestran un alto involucramiento de corte político. Al carecer de esta socialización política, o ser más bien baja, los niveles de compromiso político no pueden considerarse los necesarios para la estabilidad democrática.

Las investigaciones en el ámbito geográfico de México sobre la influencia de los hábitos comunicativos en la conformación de actitudes hacia la política y el desarrollo de prácticas dialógicas son todavía escasas. De ahí la motivación para la realización de esta investigación, centrada además en un segmento de la población menos estudiado. Los estudios previos se han centrado en el interés en analizar el proceso de la generación de opinión pública a través de los medios de comunicación en el sector de la población mayor de edad. Sin embargo, ya son diversas las investigadores que han analizado este proceso. Autores como Fernández (2005), Ibarra (2003), López (2003), Nateras (2007) y Tapia (2003) han iniciado una línea de investigación que diagnostica el estado de formación de los ciudadanos en México, tratando de identificar si estos sujetos son críticos de los procesos que se dan en la esfera pública. Por ello, el problema de investigación se puede concretar en determinar cuál es el papel de los hábitos comunicativos en la conformación de participación política y cinismo político en los preciudadanos, en el caso concreto del Área Metropolitana de Nuevo León.

\section{Preguntas e hipótesis de investigación}

El presente trabajo tiene como propósito conocer la influencia de los hábitos comunicativos, tales como el consumo de medios de comunicación y el desarrollo de prácticas tendientes a la conversación sobre política, en la conformación de las actitudes políticas, en este caso concreto la participación política y el cinismo político, en

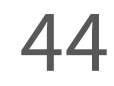


jóvenes preciudadanos menores de 18 años del área metropolitana de Monterrey, dentro del estado mexicano de Nuevo León. Para ello, se formulan las siguientes preguntas e hipótesis de investigación que se busca contestar o contrastar a partir de los resultados obtenidos

PI1. ¿Cuál es el medio de comunicación que más utilizan los jóvenes preciudadanos para enterarse de política? $\mathrm{PI}$ 2. ¿Con quiénes suelen mantener conversaciones sobre política?

HI1. La exposición a televisión refuerza el cinismo político, mientras que la exposición a medios impresos hace aumentar la participación ciudadana.

HI2. La atención a contenidos de entretenimiento contribuye a la generación de mayor cinismo político.

HI3. La conversación política, tanto interactiva u on-line como cara a cara o interpersonal, contribuye al aumento de la participación tanto política como cívica.

HI4. Bajos niveles de conversación política contribuyen al aumento del cinismo político de los jóvenes preciudadanos.

\section{Metodología}

\subsection{Participantes y procedimiento}

El estudio se realizó seleccionando una muestra representativa de los jóvenes que se encuentran inscritos en el último año escolar de la preparatoria de la zona metropolitana de Monterrey, dentro del estado de Nuevo León en México. Todos los participantes en el estudio eran estudiantes menores de 18 años o con esta edad cumplida, pero que fueran a votar por primera vez en las elecciones presidenciales celebradas en México el 1 de julio de 2012. Para determinar el tamaño de la muestra se precisó conocer los datos correspondientes al número jóvenes inscritos en el periodo escolar 2010-2011. El estado de Nuevo León contaba por ese momento con 278 preparatorias entre públicas y privadas de acuerdo al Gobierno Estatal; teniendo una población objeto de estudio de 28785 alumnos de acuerdo a cifras de la Secretaría de Educación Pública (SEP, 2010).

La muestra inicial estaba conformada por 1030 estudiantes, con un nivel de confianza del $95 \%$ y un error máximo del 3,2\%. Para realizar la selección de la muestra, en primer lugar se eligió de manera aleatoria las preparatorias separando en públicas y privadas así como de la misma manera se escogió a los grupos de último semestre en donde se aplicó el instrumento de medición. Las fechas de aplicación se dieron entre enero y febrero de 2011. Se aplicó en 11 preparatorias públicas y 12 privadas. Al finalizar la encuesta y analizar los datos recolectados se eliminaron 194 cuestionarios debido a que no correspondían a los objetivos de la investigación. Un ejemplo de ello fue que se encontraron personas de mayor edad a la que se tenía como objeto de investigación (personas con 41 años de edad). Por lo tanto la muestra final quedó conformada por 836 cuestionarios.

\subsection{Variables independientes}

Exposición a medios: se les preguntó por el tiempo que dedicaban a ver televisión, leer prensa escrita, leer prensa por Internet, escuchar la radio, leer revistas de información y navegar por Internet, siguiendo la propuesta 
de Schreiber y García (2003). Los valores de la escala oscilaban entre (1) nada y (5) bastante. Para determinar la unidimensionalidad de la escala, se realizó un análisis factorial con los diferentes reactivos que convergieron en un único componente, con un KMO =.65 $(p<.001)$ que consiguió explicar el $49 \%$ de la varianza. Sin embargo, se obtuvo un valor de Alfa de Cronbach de $\alpha=.53$, lo que permite intuir que existen patrones diferenciados de exposición a medios que impide generar una única escala. Por ello, se procedió a utilizar los diferentes ítems como independientes en los subsiguientes análisis realizados.

Atención a programas de política: mediante una escala tipo Likert de 5 puntos a fin de evaluar el grado de atención prestada a programas relacionados con la política con preguntas tales como "qué tanto atiendes a las noticias de política internacional, nacional o local en la televisión y la prensa”. Además se les cuestionó su grado de atención a programas de política tradicionales en el que se presenten debates sobre temas políticos, así como también los relacionados a la sátira política. También se presentaron cuestionamientos con respecto a los contenidos sobre política en la web y en la radio. La escala presentó unidimensionalidad, KMO $=.82(p<.001)$, y consistencia interna al obtener un valor $\alpha=.84$.

Atención a programas de entretenimiento: se utilizó la escala desarrollada por Muñiz y Maldonado (2011), la cual obtuvo una buena consistencia interna al detectarse un valor $\alpha=.74$ y un KMO $=.83(p<.001)$. Se les cuestionó sobre los niveles de atención a diversos programas de entretenimiento con preguntas como: ¿cuánta atención prestas a programas de entretenimiento en la televisión, como realities, programas de humor, telenovelas, series, películas? Además, se les preguntó también con respecto a otros medios de comunicación como la radio comercial basados en consumo de música, así como también se les cuestionó sobre su consumo de entretenimiento por Internet tales como chatear, descargar música, etc. Todas las preguntas tenían como posibles respuestas que oscilaban entre (1) nada y (5) bastante.

Conversación política interpersonal: utilizando también una escala de Likert de 5 puntos, se midió el grado de conversación sobre política de forma personal que tienen los jóvenes con diferentes grupos de la población, tales como los amigos, familia, compañeros de trabajo y/o clase, profesores y maestros, sacerdotes o líderes espirituales, además de con sus vecinos u otras personas de la calle. Para la elaboración de esta escala se tomó como referencia la utilizada en la ENCUP (2008) y se agregaron dos ítems utilizados por Shah et al. (2007). Las pruebas estadísticas arrojaron valores favorables con respecto a la unidimensionalidad de la escala (KMO =.84 $p<.001$ ), logrando que el factor explicara el $48 \%$ de la varianza, con un valor de alfa de $\alpha=.84$.

Conversación política virtual o interactiva: se buscó identificar el grado de conversación sobe política que tienen los participantes de la investigación a través de Internet. Para ello, se realizó una medición a través de una escala Likert que oscilaba entre nada (1) y bastante (5). En concreto se les cuestionó sobre qué tanto usaban el correo electrónico para organizar actividades comunitarias o sociales, discutían o conversaban sobre política o noticias que habían visto a través del correo electrónico, enviaban correos a políticos o editores de medios para dar sus opiniones, expresaban sus opiniones políticas a través de Internet, participaban en chats, fórums, blogs o cualquier espacio sobre política en Internet, siguiendo la propuesta de Shah et al. (2007). Además, se agregó un ítem preguntando si los participantes escribían sus opiniones políticas en el espacio de comentarios de noticias en periódicos digitales, siguiendo en este caso la propuesta de Muñiz, Maldonado, Leyva, López, Saldierna, Hernández y Rodríguez (2012). La escala obtuvo un valor $\alpha=.81$, lo que demuestra su consistencia interna, y un KMO $=.87$ $(p<.001)$ que explica $56 \%$ de la varianza que refleja la unidimensionalidad del indicador creado. 


\subsection{Variables dependientes}

Participación política: tomando como base un modelo de escala planteado por Kim, Wyatt y Katz (1999), se cuestionó a los participantes sobre si habían realizado o no diversas actividades tales como "Has votado en las últimas elecciones (municipales, estatales, etc.)", "Has colaborado o trabajado en alguna campaña política electoral", "Has asistido a alguna reunión o mitin político", "Has contactado o hablado con funcionarios elegidos", "Has colocado alguna manta, cartel o fotografía de protesta", "Has votado en las últimas elecciones para la mesa directiva", "Has asistido a alguna manifestación o concentración", "Asistes a reuniones de algún club o agrupación social”, "Realizas o has realizado algún tipo de trabajo voluntario", "Has trabajado en algún proyecto comunitario", "Has trabajado en apoyo de alguna causa o grupo social”, "Has asistido a alguna junta de vecinos". El análisis factorial arrojó un $\mathrm{KMO}=.77(p<.001)$ para el indicador creado que consiguió explicar el 25,14\% de la varianza, así como un valor $\alpha=.72$, lo que señala una consistencia interna aceptable de la escala.

Cinismo político: se utilizó una escala conformada por diferentes afirmaciones sobre las que se preguntó el grado de acuerdo de los participantes, mediante una escala tipo Likert que oscilaban entre nada (1) y bastante (5). En concreto, se plantearon las siguientes aseveraciones: "Las propuestas políticas solo dependen de qué tan bien le vaya al partido en los sondeos", "Los políticos están únicamente preocupados en ganar las elecciones y mantener su popularidad", "Los políticos no se ocupan de los problemas de sus áreas políticas y administrativas", "Los políticos nunca cuentan la verdad acerca de sus metas y objetivos", "Los políticos manipulan cuando presentan sus propuestas", "Nunca se sabe en qué están pensando realmente los políticos cuando hablan o actúan", "No se puede confiar en que los políticos hagan las cosas de forma correcta", "Nunca se puede confiar en lo que los políticos dicen o prometen", siguiendo la propuesta planteada por Capella y Jamieson (1997). El análisis factorial convergió en un único componente con un valor de KMO =.92 $(p<.001)$ que explica $67 \%$ de la varianza, contando además esta escala con una alta consistencia interna $(\alpha=.93)$.

\section{Análisis de resultados}

\subsection{Análisis descriptivo de las actitudes políticas}

Del total de los 836 jóvenes encuestados, la mayoría pertenecían al sexo femenino, ya que un $58 \%$ de la muestra eran mujeres (482), mientras que el $42 \%$ eran hombres (354), y hubo 7 casos perdidos, en los que el encuestado no contestó esa pregunta. Analizando el tipo de escuela a la que acudían, en su mayoría la muestra pertenecía a una escuela pública con 490 jóvenes que corresponde al 58,6\%, mientras que el 41,4\% (346 casos) cursaba en escuela privada. Con respecto a la edad, se obtuvo en promedio una $M=16,64(D E=0,67)$, detectándose que en su mayoría los participantes tenían 17 años (46,2\%). Le seguían los participantes de 16 años con un 43,2\% que representaban 361 casos en la encuesta, mientras que los de 18 y 15 años tenían el 9,6\% y 1, 1\% de presencia respectivamente.

Con respecto al consumo de medios, se encontró evidencia de que los medios que más consumían los jóvenes era el Internet $(M=4,35, D E=.98)$, seguido por la televisión $(M=3,91, D E=1,04)$. Por otro lado, la prensa digital $(M=2,75, D E=1,4)$, la radio $(M=2,89, D E=1,12)$ y las revistas informativas $(M=2,57, D E=1,0)$ tuvieron poca presencia como medios utilizados por los jóvenes encuestados. Finalmente, el medio que menos utilizaban fue la prensa 
escrita $(M=2,49, D E=.96)$. En referencia al hábito comunicativo de la conversación sobre política, se encontró que las personas con las que más hablaban de política era con los familiares $(M=2,61, D E=1,09)$, seguido de profesores y maestros $(M=2,37, D E=1,07)$, compañeros de clase $(M=2,00, D E=0,98)$ y amigos $(M=1,95, D E=1,04)$. Es decir, se habla con el círculo cercano, con el que conviven día a día, pero no con otras personas como vecinos $(M=1,66$, $D E=0,89)$ o sacerdotes y líderes espirituales $(M=1,30, D E=0,64)$. Finalmente, era bajo el nivel de conversación con personas con ideas distintas a las suyas $(M=1,92, D E=1,07)$, pero también con personas que tienen ideas similares $(M=1,92, D E=1,04)$, lo que confirma el bajo nivel de debate político mantenido.

Tabla 1. Estadísticos descriptivos de las actitudes políticas

\begin{tabular}{|l|c|c|c|c|c|}
\hline & Mín. & Máx. & M & DE & \% \\
\hline Participación política & 1 & 3,86 & 1,32 & 0,57 & 16 \\
\hline Participación cívica & 1 & 5 & 2,06 & 1,29 & 19 \\
\hline Cinismo político & 1 & 5 & 3,55 & 1,01 & 75,5 \\
\hline
\end{tabular}

Nota: $N=834$. Los valores incluidos en la columna del porcentaje representa el tamaño del grupo que se sitúa por encima de la media teórica (3).

Fuente: elaboración propia.

Con respecto a las variables relacionadas a las actitudes políticas, se procedió a generar indicadores que midieran la participación desagregando tanto en participación política como cívica así como el cinismo político. Se puede observar en la tabla 1, que con respecto a la participación política no se presentaron niveles elevados de participación política $(M=1,32, D E=0,57)$, con respecto a la participación cívica, el resultado tampoco supera la media teórica pero se puede evidenciar que se tiene mayor presencia que la participación política ( $M=2,06, D E=1,29)$. En referencia al cinismo político, se encuentra un sentimiento de desafecto respecto a lo referente a la política $(M=3,55, D E=1,01)$, única actitud que se encuentra por encima de la media teórica (3), así como que el 75,5\% de los participantes en el estudio presentan cinismo político.

\subsection{Factores explicativos de la participación ciudadana}

En la segunda fase del análisis de resultados, se buscó determinar qué variables explicaban los diferentes componentes de la participación política, participación cívica y del cinismo político. Para ello, se realizaron diferentes análisis de regresión lineal múltiple jerárquica, en la opción de pasos sucesivos, para cada una de las variables dependientes contempladas. En el primer bloque se utilizaron las variables de control relacionadas a los aspectos socio-demográficos tales como el sexo del encuestado, la edad, el nivel de ingresos de la familia, así como el tipo de escuela al que pertenecen (modelo 1).

En el segundo modelo se agregaron las variables relacionadas a su exposición a los diferentes medios de comunicación como la televisión, la prensa escrita, la prensa digital, la radio, las revistas de información así como 
el Internet. En el siguiente paso se agregaron los niveles de atención a los diversos contenidos consumidos en los medios ya sean atención a contenidos de política así como los de entretenimiento (modelo 3). Finalmente en el modelo 4 fueron añadidas las variables de conversación política interpersonal e interactiva. En todos los casos se verificó que las variables cumplieran el supuesto de independencia con respecto a la variable dependiente. Para ello, se utilizó la prueba de Durbin-Watson que requiere que su valor oscile entre el límite mínimo 1,5 y el límite máximo 2,5.

Tabla 2. Variables explicativas de la participación política

\begin{tabular}{|c|c|c|c|c|}
\hline & \multicolumn{4}{|c|}{ Modelo } \\
\hline & 1 & 2 & 3 & 4 \\
\hline \multicolumn{5}{|l|}{ Variables socio-políticas } \\
\hline Sexo del encuestado & .004 & .018 & .018 & .042 \\
\hline Edad del encuestado & .045 & .053 & .053 & .048 \\
\hline Nivel de ingresos mensual de tu familia: & .080 & .066 & .066 & .046 \\
\hline Tipo de Escuela & -.009 & -.016 & -.016 & -.017 \\
\hline \multicolumn{5}{|l|}{ Exposición mediática } \\
\hline Televisión & & -.053 & -.053 & .018 \\
\hline Prensa escrita & & $.097^{\star}$ & $.097^{\star}$ & .033 \\
\hline Prensa digital por Internet & & $.106^{\star}$ & $.106^{\star}$ & -.042 \\
\hline Radio & & -.048 & -.048 & .057 \\
\hline Revistas de información & & $.116^{\star}$ & $.116^{\star}$ & .018 \\
\hline Internet & & .030 & .030 & .051 \\
\hline \multicolumn{5}{|l|}{ Atención a contenidos } \\
\hline Atención política & & & $.211^{\star \star \star}$ & .054 \\
\hline Atención entretenimiento & & & -.091 & -.064 \\
\hline \multicolumn{5}{|l|}{ Conversación política } \\
\hline Conversación personal & & & & $.188^{\star \star \star}$ \\
\hline Conversación interactiva & & & & $.135^{\star \star}$ \\
\hline$\Delta R^{2}$ & .009 & .052 & .031 & .047 \\
\hline$R^{2}$ & .009 & .060 & .092 & .138 \\
\hline
\end{tabular}

Nota: $N=834$. La variable sexo del encuestado se recodificó como dummy, donde 1 significa ser mujer. Con respecto a la escuela se hizo lo mismo donde 1 significaba escuela privada. Los resultados fueron estadísticamente significativos a un nivel de confianza de ${ }^{\star} p<.05 ;{ }^{\star \star} p<.01,{ }^{\star \star \star} p<.00$.

Fuente: elaboración propia. 
Al hacer el análisis de las variables explicativas de la variable dependiente constituida por la participación política, se observó que ninguna de las variables socio-políticas explicaba este tipo de participación (ver tabla 2). En el primer modelo tan solo se explica el $0,9 \%$ de la varianza, aunque como se ha indicado ninguna variable logró impactar en la participación política de los participantes. Al incorporar las variables relacionadas a los medios de comunicación en el segundo modelo, se detectó que el consumo de los contenidos en medios escritos constituyó la variable que más se relacionaba con el conocimiento político; tanto la exposición a prensa escrita $(\beta=.097, p<.001)$ a prensa digital $(\beta=.106, p<.05)$, como a las revistas de información $(\beta=.116, p<.05)$ explicaban la participación política. La varianza explicada en este segundo modelo fue del $5,2 \%\left(R^{2}=.060\right)$.

En el siguiente paso, constitutivo del tercer modelo, se adicionan las variables relacionadas a la atención a contenidos. En la ecuación obtenida se observó que, si bien la exposición a medios escritos influía en la participación política, los contenidos de los medios de comunicación que influían eran centrados en temas de política $(\beta=.211$, $p<.001)$. Este tercer modelo calculado logró explicar el 3,15\% del total de la varianza $\left(R^{2}=.092\right)$. Finalmente, en el último paso o modelo generado se introdujeron las variables sobre conversación. Se observó en la ecuación resultante que todas las anteriores variables que explicaban el modelo desaparecían de la ecuación, manteniéndose únicamente como variables explicativas la conversación política interpersonal $(\beta=.188, p<.001)$ y la conversación política interactiva $(\beta=.135 p<.01)$. En este modelo se consiguió explicar el $4,7 \%$ de la varianza $\left(R^{2}=.138\right)$.

El análisis de la ecuación explicativa de la variable dependiente: participación cívica, arrojó cambios claros con respecto a la participación política de los mismos participantes (ver tabla 3). En ese sentido, las variables socio-políticas consiguieron explicar en el primer modelo un $4 \%$ de la varianza total. Se observó que el sexo ( $\beta=.120$, $p<.05)$ y el ingreso familiar $(\beta=.150, p<.001)$ explicaban la participación cívica de los encuestados. Por lo que el ser mujer con un nivel de ingresos alto, explicaba el hecho de que los participantes estuvieran más involucrados en acciones tales como apoyo a la comunidad, o asistir a juntas de vecinos.

En el segundo modelo, al añadir las variables de exposición mediática la ecuación aumentaba un $5,8 \%$ la varianza explicada $\left(R_{\text {total }}^{2}=.097\right.$ ). En concreto, se observó que la variable consumo o exposición a televisión ( $\beta=-.112$, $p<.01)$ impactaba en el nivel de participación cívica, pero en sentido negativo. Es decir, un menor consumo de televisión generaba mayores niveles de participación cívica. Además, también se detectó que la exposición o consumo de la prensa escrita $(\beta=.126, p<.01)$, de las revista de información $(\beta=.088, p<.05)$ y navegar asiduamente por el Internet $(\beta=.111, p<.01)$ explicaban la participación cívica en sentido positivo. Es decir, cuanta más exposición se daba a estos medios de comunicación, mayores eran los niveles de participación cívica encontrados en los jóvenes. 
Tabla 3. Variables explicativas de la participación cívica

\begin{tabular}{|c|c|c|c|c|}
\hline & \multicolumn{4}{|c|}{ Modelo } \\
\hline & 1 & 2 & 3 & 4 \\
\hline \multicolumn{5}{|l|}{ Variables socio-políticas } \\
\hline Sexo del encuestado & $.120^{*}$ & $.132^{\star \star \star}$ & $.184^{\star \star \star}$ & $.164^{\star \star \star}$ \\
\hline Edad del encuestado & .002 & .008 & .009 & .002 \\
\hline Nivel de ingresos mensual de tu familia: & $.150^{\star \star \star}$ & $.129^{\star \star}$ & $.117^{\star \star}$ & $.105^{\star \star}$ \\
\hline Tipo de escuela & .057 & .049 & .044 & .047 \\
\hline \multicolumn{5}{|l|}{ Exposición mediática } \\
\hline Televisión & & $-.112^{\star \star}$ & $-.107^{\star}$ & $-.086^{*}$ \\
\hline Prensa escrita & & $.126^{\star \star}$ & .065 & .040 \\
\hline Prensa digital por Internet & & .028 & -.014 & -.052 \\
\hline Radio & & .049 & .032 & .046 \\
\hline Revistas de información & & $.088^{\star}$ & .042 & .020 \\
\hline Internet & & $.111^{\star \star}$ & $.132^{\star \star}$ & $.129^{\star \star}$ \\
\hline \multicolumn{5}{|l|}{ Atención a contenidos } \\
\hline Atención política & & & $.239^{\star \star \star}$ & .093 \\
\hline Atención entretenimiento & & & -.055 & -.033 \\
\hline \multicolumn{5}{|l|}{ Conversación política } \\
\hline Conversación personal & & & & $.145^{\star *}$ \\
\hline Conversación interactiva & & & & $.164^{\star \star \star}$ \\
\hline$\Delta R^{2}$ & .040 & .058 & .038 & .043 \\
\hline$R^{2}$ & .040 & .097 & .135 & .178 \\
\hline
\end{tabular}

Nota: $N=834$. La variable sexo del encuestado se recodificó como dummy, donde 1 significa ser mujer.

Con respecto a la escuela se hizo lo mismo donde 1 significaba escuela privada. Los resultados fueron estadísticamente significativos a un nivel de confianza de ${ }^{\star} p<.05 ;{ }^{\star \star} p<.01,{ }^{\star \star \star} p<.001$

Fuente: elaboración propia.

En el siguiente paso se agregaron las variables referentes a la atención a contenidos de los medios de comunicación. En este modelo, la exposición a la prensa escrita y navegar por el Internet dejaron de explicar en la ecuación, pero se incorporó como variable explicativa la atención a contenidos de política en los medios $(\beta=.239$, $p<.001)$. Esta variable logró aportar $3,8 \%$ de la varianza $\left(R_{\text {total }}^{2}=.135\right)$. Finalmente, en el cuarto modelo la conversación política interpersonal $(\beta=.145, p<.01)$ y la conversación interactiva $(\beta=.164, p<.001)$ también lograron explicar la participación cívica. Además, al introducirse estas variables la atención a contenidos de política dejó de aportar a la participación cívica. Este modelo logra explicar el 4,3\% $\left(R_{\text {total }}^{2}=.178\right)$. 


\subsection{Factores explicativos del cinismo político}

\section{Tabla 4. Variables explicativas del cinismo político}

\begin{tabular}{|c|c|c|c|c|}
\hline & \multicolumn{4}{|c|}{ Modelo } \\
\hline & 1 & 2 & 3 & 4 \\
\hline \multicolumn{5}{|l|}{ Variables socio-políticas } \\
\hline Sexo del encuestado & .071 & $.079^{\star}$ & $.084^{\star}$ & .074 \\
\hline Edad del encuestado & .008 & .012 & .013 & .010 \\
\hline Nivel de ingresos mensual de tu familia: & $.159^{\star \star \star}$ & $.157^{\star \star \star}$ & $.153^{\star \star \star}$ & .150 \\
\hline Tipo de escuela & .007 & -.001 & -.002 & -.002 \\
\hline \multicolumn{5}{|l|}{ Exposición mediática } \\
\hline Televisión & & -.005 & -.026 & -.014 \\
\hline Prensa escrita & & $.129^{\star \star}$ & $.118^{\star \star}$ & $.109^{\star}$ \\
\hline Prensa digital por Internet & & -.070 & -.077 & 089* \\
\hline Radio & & -.022 & -.034 & -.035 \\
\hline Revistas de información & & -.010 & -.019 & -.018 \\
\hline Internet & & .078 & .074 & .071 \\
\hline \multicolumn{5}{|l|}{ Atención a Contenidos } \\
\hline Atención política & & & .032 & -.029 \\
\hline Atención entretenimiento & & & .038 & .049 \\
\hline \multicolumn{5}{|l|}{ Conversación política } \\
\hline Conversación personal & & & & $.127^{\star}$ \\
\hline Conversación interactiva & & & & -.017 \\
\hline$\Delta R^{2}$ & .022 & .105 & .116 & .034 \\
\hline$R^{2}$ & .022 & .125 & .241 & .275 \\
\hline
\end{tabular}

Nota: $N=834$. La variable sexo del encuestado se recodificó como dummy, donde 1 significa ser mujer.

Con respecto a la escuela se hizo lo mismo donde 1 significaba escuela privada. Los resultados fueron estadísticamente significativos a un nivel de confianza de ${ }^{\star} p<.05 ;{ }^{\star \star} p<.01,{ }^{\star \star \star} p<.001$.

Fuente: elaboración propia.

Finalmente, se generó un análisis de regresión lineal múltiple para estudiar las variables explicativas del nivel de cinismo político presentado por los participantes (ver tabla 4). El primer modelo o bloque constituido por las 
variables socio-demográficas consiguió explicar el 1,9\% de la varianza. Al respecto, la variable relativa al nivel de ingresos fue estadísticamente significativa $(\beta=.159, p<.001)$, por lo que un nivel de ingresos elevado explicaba el aumento en el cinismo político. Al introducir al modelo las variables de exposición mediática (modelo 2), se generó una explicación del 4,9\% de la varianza $\left(R_{\text {total }}^{2}=.105\right)$. En este modelo se comenzó a explicar el sexo del encuestado $(\beta=.079, p<.05)$ y se mantuvo la explicación del nivel de ingresos $(\beta=.157, p<.001)$. Además, apareció el impacto que tiene el consumo de la prensa escrita $(\beta=.129, p<.01)$. Esto es, ser mujer con un nivel elevado de ingresos y consumir prensa escrita son factores que contribuyen a generar cinismo político.

En el tercer modelo, se incorporaron las variables acerca de la atención a contenidos de política y de entretenimiento en los medios de comunicación. La varianza mostró un cambio, aumentando en un 5,8 el porcentaje explicado $\left(R_{\text {total }}^{2}=.116\right)$. En este modelo seguían explicando el sexo $(\beta=.084, p<.05)$, el nivel de ingresos $(\beta=.153$, $p<.001)$ y el consumo de prensa escrita $(\beta=.118, p<.01)$. Sin embargo, ni la atención a contenidos de política en medios ni a programas de entretenimiento consiguieron explicar el cinismo político de los participantes en el estudio. Finalmente, en el cuarto modelo explicativo del cinismo político, las variables sexo y nivel de ingresos dejaron de explicar. Siguió impactando en el cinismo el consumo de prensa escrita $(\beta=.109, p<.05)$, a la que se unió además el consumo de prensa digital $(\beta=.089, p<.05)$. En cuanto a la conversación sobre política, cuyas variables fueron incorporadas en este modelo, solo logró impactar la interpersonal $(\beta=.127, p<.05)$. Este modelo logra explicar el 5,8\% del total de la varianza $\left(R_{\text {total }}^{2}=.034\right)$.

\section{Discusión y conclusiones}

El presente estudio buscaba conocer la influencia que los hábitos comunicativos, tales como el consumo de medios de comunicación o la conversación sobre política, tienen en la conformación de la participación política y el cinismo político entre jóvenes menores de 18 años, también llamados en el estudio como preciudadanos. Esto en el escenario de las elecciones presidenciales de 2012 en la zona metropolitana de Monterrey en el estado mexicano de Nuevo León.

Con respecto a la primera pregunta de investigación, que se planteaba: ¿cuál es el medio de comunicación que más utilizan los jóvenes preciudadanos para enterarse de política? Se encontró evidencia de que los jóvenes tienen elevados niveles de consumo y exposición a medios de comunicación, siendo el Internet y la televisión a los que más se exponen, mientras que hay muy poca influencia de la prensa escrita. En referencia a la atención a los contenidos, se encontró que una gran parte de los jóvenes no están interesados en información política, puesto que la mayoría de los encuestados afirmaban no ver ningún programa sobre este tema en la televisión, en un nivel similar a los que aseveraban no navegar en ningún blog o página que contuviera contenidos políticos. Así, solo los utilizan con fines de diversión. Además, se halló que para lo que más usan el Internet es para navegar, bajar música y chatear, mientras que la televisión la utilizan para ver series de televisión.

En ese sentido, el papel de los medios como herramienta diseminadora de información sobre el mundo político no ejerce ese rol en los jóvenes, ya que ellos están filtrando la información que consumen, con lo cual pudiera gestar una generación apática y desconectada con respecto a la política y el bienestar social de su entorno. Poniendo en evidencia la falta de interés e indiferencia que los preciudadanos tienen ante temas relacionados con la política, solo piensan en diversión y en pasarla bien. Lo que podría considerarse como un hecho típico de su edad, lo 
importante a recalcar es que están a un paso de enfrentarse a la toma de decisiones que repercutirán en su futuro, no solo respecto al ámbito político sino también a su preparación profesional para su vida adulta, debido a que están en el último semestre de preparatoria, tienen que enfrentarse a la decisión de elegir profesión y no están gestando ningún tipo de compromiso ni político ni cívico, lo que lleva a una generación de individualismo que solo piensa en su bienestar personal pero sin mucho esfuerzo.

Este comportamiento puede afectar la cohesión social propiciando un escenario ideal para una desestabilidad social o simplemente que no exista una crítica al sistema permitiendo que el desempeño del gobierno no sea el ideal en una democracia.

Si los jóvenes no están interesados en los contenidos de política divulgados a través de los medios de comunicación, surge la interrogante sobre si la información política con la que cuentan puede provenir de otros mecanismos comunicativos. En relación con ello, la segunda pregunta de investigación planteaba: ¿con quiénes suelen mantener conversaciones sobre política? Los participantes en el estudio afirmaron que no hablan de política con personas que compartan sus mismas ideas, pero tampoco con personas de diferentes ideas, lo cual muestra que no están confrontando sus ideas con respecto a la política con nadie. Esto es, no buscan hablar del tema con individuos que tengan una ideología u opinión similar, pero tampoco lo hacen con los que difieren de ellos, lo cual genera que no estén enriqueciendo su conocimiento político, ya que el debatir sobre lo que se piensa permite generar cambios en las opiniones o hacerlas más fuertes. Es importante recalcar que fue sorprendente detectar que siguen viendo a la familia y a los profesores como personas de confianza y de autoridad como para hablar con ellos de temas políticos, ya que la mayoría afirmó hablar de política con sus familiares y con los profesores, son las personas con las que más hablan sobre esos temas. Este resultado refleja la existencia de una democracia inmadura que no ha evolucionado y sigue viendo como autoridades educativas estos dos grupos de población.

Los resultados obtenidos en el estudio permiten vislumbrar las condiciones en que se generan las actitudes políticas de los preciudadanos, al menos en el caso de la zona metropolitana de Monterrey, en México. En la primera hipótesis de investigación se planteaba que la exposición a la televisión refuerza el cinismo político, mientras que la exposición a medios impresos hace aumentar la participación ciudadana. Los resultados derivados de la investigación no permiten probar esta hipótesis, ya que no se encontró evidencia estadística de la trascendencia del consumo de la televisión en la gestación de esta actitud. Este resultado constituye una gran aportación, en tanto que contradice lo evidenciado por autores previamente, como es el caso de Robinson (1976). Así mismo, contradice la propuesta de Newton (1999) sobre el impacto de la televisión en los sentimientos negativos hacia la política, los ciudadanos que más televisión consumen son los que más apáticos al sistema son, y por tanto con niveles de aversión elevados con respecto al compromiso cívico y político (Capella \& Jamieson, 1997; de Vreese, 2005; Maiers, 2000). Finalmente, con respecto a la conversación política interpersonal y la conversación interactiva también lograron explicar la participación cívica. Además, al introducirse estas variables la atención a contenidos de política dejó de aportar a la participación cívica. Este resultado explica el hecho de que las mujeres que más conversan sobre política son las más involucradas en participar en acciones relacionadas al trabajo comunitario así como en apoyar en causas sociales. Así mismo, son las que más emprenden acciones relacionadas a la participación política, lo cual concuerda con lo evidenciado por Rojas (2006) y Newton (2006).

En referencia a la segunda hipótesis de investigación, que plantea que la atención a contenidos de entretenimiento contribuye a la generación de mayor cinismo político, tampoco se pudo demostrar estadísticamente

$$
54
$$


esta relación. En el estudio se encuentra evidencia que refuta lo propuesto teóricamente, los contenidos de entretenimiento no influyen en la generación de la apatía. Lo que está impactando en la formación del cinismo político en los ciudadanos es el consumo de prensa tanto escrita y digital, lo cual contradice lo propuesto por Norris (2000), Newton (1999). Los que prestan más atención a contenidos sobre política son también los más apáticos, son cínicos políticamente pero con información.

Por su parte, la tercera hipótesis de investigación planteaba que la conversación política, tanto interactiva u online como cara a cara o interpersonal, contribuye al aumento de la participación tanto política como cívica. Los resultados obtenidos en los diferentes análisis de regresión múltiple realizados permitieron determinar que los niveles de conversación cara a cara y la conversación interactiva impactan de manera considerable en el aumento de esta actitud, fomentando el incremento de activismo político en los jóvenes preciudadanos. Así mismo, los niveles de participación cívica también son alimentados por la conversación interpersonal y la conversación en línea. Ello confirma el hecho de que los niveles de conversación sobre política elevados favorecen la procreación de altos niveles de integración social y movilización evidenciada a través de la participación política y cívica, en la misma línea a lo detectado por estudios previos (e.g. McLeod et al., 1999; Rojas, 2006).

Finalmente, en la cuarta hipótesis de investigación se planteaba que unos bajos niveles de conversación política contribuyen al aumento del cinismo político de los jóvenes preciudadanos. Sin embargo, se ha observado en los resultados obtenidos que la conversación política interpersonal genera más bien un aumento del sentimiento de desafección política, contradiciéndose de esta manera la evidencia teórica previa acerca de la influencia positiva que tienen estas acciones en la generación de movilización política (Lee, Shah, \& McLeod, 2012; McLeod et ál., 1999). Esto es, entre mayores sean los niveles de conversación sobre política que tengan los preciudadanos, mayor será el cinismo político. Lo cual, recordando que con quienes más hablan de política son sus padres, podría cuestionar si la apatía y la desafección hacia la política sea una actitud heredada a través de las generaciones.

La comprobación de las hipótesis a través del uso de regresiones jerárquicas múltiples ha posibilitado encontrar evidencias relevantes para la explicación de la conformación de diferentes actitudes políticas de los preciudadanos que claramente contradicen supuestos teóricos previos, al menos en el caso de los jóvenes del área metropolitana de Monterrey, donde se realizó el estudio.

Se evidenció que, con respecto a la exposición mediática, la televisión no está ejerciendo ninguna influencia en la generación de actitudes políticas como la participación o el cinismo político, así como también la evidencia de que el uso de la prensa escrita es casi inexistente en este segmento de la población. Sin embargo, fue contradictorio encontrar esa relación de explicación entre actitudes de desmovilización como el cinismo político y el consumo de prensa escrita, cuando este tipo de medio está más relacionado a fomentar la movilización política.

En estos resultados se puede apreciar que, más que los medios, lo que realmente está contribuyendo en la generación de actitudes políticas son los niveles de conversación política, tanto cara a cara como de forma interactiva. Este hábito refuerza las actitudes como la participación política y la participación cívica. Con lo cual, queda demostrado que la formación de los preciudadanos no es a través de los medios de comunicación. El proceso en el que se van formando las actitudes hacia la política denominada socialización política se limita al entorno familiar y de escuela, su contexto inmediato. Si los padres son apáticos, desafectos y desinteresados en la política este sentimiento es transmitido a través de las opiniones hacia sus hijos, heredando esa actitud. Esto por el rol de influencia de poder que tienen los padres a esa edad en los hijos. Es la etapa en la que transmiten los valores que repercutirán 
en el comportamiento de sus hijos en la vida adulta en todos los ámbitos incluidos el cívico-político, además de ser los ejemplos a seguir (Niemi y Hepburn, 1995; Funes, 1994). Por tanto, las acciones y formas de pensar de los padres tienen un impacto decisivo en la formación de ciudadanos. En ese sentido, de acuerdo a datos de Parametría, obtenidos en 2013, el $57 \%$ de los participantes en un estudio a nivel nacional en México, consideraban que es vital el respeto a los padres, de ahí la evidencia de lo relevante que es para los hijos la opinión de sus progenitores.

Por tanto, es necesario generar mecanismos en los que se propicie el involucramiento de más elementos formadores de ciudadanos. Es importante analizar los contenidos sobre política que se están presentando, tal vez estos no están enfocados en formar ciudadanos más jóvenes. Así como tampoco se están trasmitiendo contenidos que impacten, también, a los más grandes, o que promuevan la generación de un involucramiento con niveles de compromiso elevado en cuestiones de ayuda social y de participación política. Y que de alguna manera a través del círculo de trasmisión de información evidenciado lleguen a los preciudadanos. Otro elemento clave a considerar son las acciones que las escuelas deben emprender para que se enriquezca la formación de ciudadanos desde edades tempranas, por ello, es importante revisar los planes de estudios. Es posible que las Instituciones educativas se estén limitando en la formación de recursos humanos para el ámbito profesional y laboral y no cómo elementos críticos y necesarios de un sistema político democrático. La escuela y los estímulos generados a través de la conversación con los padres repercutirán en el comportamiento político de los hijos en la vida adulta (Verba et al., 1995).

\section{Referencias}

1. Abundis, F. (2007). Los medios de comunicación. AMAl, 13, $42-45$.

2. Anduiza, E. \& Bosch, A. (2004). Comportamiento Político y Electoral. Barcelona: Ariel.

3. Anduiza, E., Cantijoch, M., Colombo, C., Gallego, A. \& Salcedo, J. (2010). Los usos políticos de Internet en España. Revista Española de Investigaciones Sociológicas, 129, 133-146.

4. Avery, J. (2009). Videomalaise or Virtuous Circle. The Influence an the New Media in Political Trust. The International Journal of Press/Politic., 14, pp. 410-433.

5. Capella, A. \& Jamieson, K. (1997). Spiral of Cynicism: The Press and the Public Good. New York: Oxford Unviersity Press.

6. Conell, R. (1971). The Child's Construction of Politics. Carlton, Australia: Melburne University Press.

7. Conway, M. (1986). La participación política en Estados Unidos. México: Ediciones Gernika.

8. Cordourier, C. (2008). Educación para la participación ciudadana. AZ Revista de Educación y Cultura, 8 , pp. $22-25$

9. Coto, M. (1970). La agencia de la niña y el niño en la condición pre-ciudadana. Actualidades Investigativas en Educación, 12(2),11-19.

10. de Vreese, C. (2005). The Spiral of Cynisim Reconsidered: the Mobilizing Function of News. European Journal of Communication, 20,. 283-301.

11. ENCUP. (2008). Encuesta nacional sobre cultura política y prácticas ciudadanas. México: Secretaría de Gobernación. 
12. Fernández, A. (2005). Infancia, adolescencia y política en México. México D.F.: Porrúa / Instituto Electoral del Distrito Federal.

13. Funes, M. (1994). La dimensión social del altruismo. Sociedad y utopía, 4, 192-204.

14. García-Luengo, O. (2002). La (Im)pertenencia de las teorías del malestar mediático en el caso español, una primera aproximación empírica. Revista Iberoamericana de Comunicación, 3, 89-106.

15. García, G. y Micco, S. (1997). Hacia una Teoría del Pre-ciudadano. Bogotá: Uriel-UNICEF.

16. García-Luengo, O. \& Maurer, M. (2009). A Virtuous Cirlce for All? Media Exposure and political Trust in Europe. Confines, 9, 39-48.

17. Gómez, S., Tejera, H., \& Aguilar, J. (2012). La cultura política de los jóvenes en México. México D.F.: El Colegio de México.

18. Guerrero, M. (2006). Medios de comunicación y la función de transparencia. México: Instituto Federal de Acceso a la Información.

19. Holtz-Bacha, C. (1990). Videomalaise Revisited: Media Exposure and Political Alienation in West Germany. European Journal of Communication, 5(1), 73-85.

20. Ibarra, A. (2003). Televisión y socialización política de escolares de la zona metropolitana de Guadalajara. Jalisco: Universidad de Guadalajara.

21. IFE. (2002). Programa Estratégico de Educación Cívica. México: Instituto Federal Electoral.

22. IMJ. (2005). Encuesta Nacional de la Juventud. México: Centro de Investigaciones y Estudios sobre Juventud.

23. INEGI. (2010). Conteo Nacional de Población y Vivienda. México: Instituto Nacional de Estadística y Geografía.

24. Kim, J., Wyatt, R., \& Katz, E. (1999). New Talk Opinion, Participation: The Part Played by Conversation in Deliberative Democracy. Political Communication, 16(4), 361-385.

25. Lang, K., \& Lang, G. (1966). The Mass Media and Voting. En B. Berelson \& M. Janowitz. Reader in public opinion and communication (pp. 55-472). New York: Free Press.

26. Lee, N., Shah, D., \& McLeod (2012). Processes of Political Socialization A Communication Mediation Approach to Youth Civic Engagement. Communication Research, 40(5), 669-697.

27. López, G. (2003). Televisión y socialización política de los niños de Monterey y Juchitán (Tesis de Maestría). Tecnológico de Monterrey, Monterrey.

28. López, F. (2007). Metodología participativa de la enseñanza universitaria. Colección Universitaria. Madrid: Editorial Norcea.

29. Maiers, J. (2000). Politikverdrossenheith in Deutschland Dismensionen-Determinanten konsequenzes. Lesket Budrich: Op Landen.

30. Martínez, S., \& Salcedo, A. (2006). Diccionario Electoral INEP. México: Instituto Nacional de Estudios Politicos AC.

31. McLeod, J., Sheufele, D., \& Moy, P. (1999). Community, Communication, and Participation: The Role of Mass Media and Interpersonal Discussion in Local Political Participation. Political Communication, 16(3), 315-336.

32. Muñiz, C., \& Maldonado, L. (2011). Entre la movilización y el malestar mediático. Impacto de las prácticas comunicativas en las actitudes políticas de los jóvenes. Perspectivas de la Comunicación, 4(2), 32-54.

\section{7}


33. Muñiz, C., Maldonado, L., Leyva, O., López, R., Saldierna, A., Hernández, T., \& Rodríguez, E. (2012). Habitos comunicativos y sofisticación política. En Carlos Muñiz (Ed.), Comunicación Política y Ciudadanía (pp. 237253). México: Fontamara Editores.

34. Nateras, J. (2007). Información política en estudiantes de primariay secundaria: aspectos socioeconómicos y exposición a medios de comunicación: los inicios de la socialización política. Revista Mexicana de Investigación Educativa, 12(35), 1357-1403.

35. Newton, K. (1999). Mass Media Effects: Mobilization or Media Malaise? British Journal of political science, 29(4), 577-599.

36. Newton, K. (2006). May the Weak Force Be With You: The Power of the Mass Media in Modern Politics. European Jorunal of Political Research, 45(2), 209-234.

37. Niemi, R., \& Hepburn, M. (1995). The Rebirth of Political Socialization. Perspectives on Political Science, 24(1), $1-18$.

38. Niemi, H. (1999). Moving Horinzons in Education. International Transformations and Challenges of Democracy. Helsinki: Deparment of Education University Press.

39. Norris, P. (1996). Does Television Erode Social Capital? A Replay to Putnam. Political Science and Politics, 29(3), 474-480.

40. Norris, P. (2000a). A Virtuous Circle. Political Communications in Postindustrial Societies. Cambridge: Cambridge University Press.

41. Norris, P. (2000b). Impact of Television on Civil Malaise. En R. Putnam, \& S. Phar (Eds.). Disaffected Democrats: What's Troubling the Trilateral Democracies (pp. 231-251). Princenton, NJ: Princeton University Press.

42. Parametría (2013). Encuesta Nacional en Vivienda. México: Parametría.

43. Pasquino, G. (1994). Participación política, grupos y movimientos. En G. Pasquino, S. Bartolini, M. Cotta, \& L. Molinero. (Eds.). Manual de Ciencia Política. Madrid: Alianza Universiad Textos.

44. Prior, M. (2005). News vs. Entertainment: How Increasing Media Choice Widens Gaps in Political Knowledge and Turnout. American Journal of political sciences, 49(39), 577-592.

45. Robinson, M. (1976). Public Affair Television and the Growth of Political Malaise. The Case of the Selling the Pentagon. American Political Science Review, 70(2), 409-432.

46. Rojas, H. (2006). Comunicación, participación y democracia. Universitas Humanistica, 62(62), 109-149.

47. Sartori, G. (2005). Homovidens: la sociedad teledirigida. México: Taurus.

48. Schreiber, D., \& García, O. (2003). ¿Videomalestar o círculo virtuoso? Una primera aproximación empírica a la exposición mediática y el compromiso político en España y Alemania. Política y Sociedad, 41(4), 131-143.

49. Schmitt-Beck, R., \& Mckenrodt, C. (2010). Social Networks and Mass Media as Mobilizers and Dmobilizers: a Study of Turnout at German Local Election. Electoral Studies, 24(3), 392-404.

50. SEP. (2010). Estadísticas e indicadores educativos por entidades. México: Secretaría de Educación Pública.

51. Shah, D., McLeod, J., \& Yoon, S. (2001). Communication, Context and Community: an Explorations of Print, Broadcast and Internet Influences. Communication Research, 28(4), 464-506.

52. Shah, D., Cho, J., Nah, S., Gotlieb, M., Lee, N., Scholl, R., Hwang, H., \& McLeod, D. (2007). Campaign Ads, Online Messaging and Participation: Extending the Communication Mediation Model. Journal of Communication, 57, 676-703. 
53. Sirkku, K. (2009). Participación cívica y producción mediática de los jóvenes: voz de la juventud. Comunicar, 16(32), 181-196.

54. Stromback, J., \& Shehata, A. (2010). Medial Malise or a Virtuous Circle? Exploring the Causal Relationships Between Media Exposure, Political News Attention and Political Interest. European Journal of Political Research, 49(5), 575-597.

55. Tapia, E. (2003). Socialización política y educación cívica en los niños. Querétaro: Instituto Mora.

56. Torgal, M. (2000). La desafección democrática en las nuevas democracias: sus orígenes y consecuencias. En R. Máiz Suárez (Ed.). Construcción de europa, democracia y globalización (pp. 1165-1208). Santiago de Compostela: Universidad de Santiago de Compostela.

57. Verba, S., Schlozman, K., \& Brady, H. (1995). Voice and Equality Civic. Civic Voluntarism in American Politics. Cambridge: Harvard University Press.

58. Voltmer, K., \& Schmitt-Beck, R. (2007) The Mass Media in Third-Wave Democracies: Gravediggers or Seedsmen of Democratic Consolidation? Democracy, Intermediation, and Voting in Four Continents (pp. 75134). Oxford: Oxford Univerisity.

59. Voltmer, K. (2007). Mass Media and Politcal Communication in a New Democracy. London: Routledge. 